at the time when the intestines were accused of everything during childhood.

The treatment applied was inunction with Credés ointment and treatment of the accompanying nephritis.

This should only be considered a preliminary report. In the near future we hope for more detailed reports from pnysicians who have hospitals near at hand with better opportunities for closer examinations and clinical investigations.

\section{FRACTURE OF PATELLA.}

RESTORATION OF FUNCTION IN SPITE OF NON-TREATMENT. J. B. CUTTER, M.D.

Surgeon in Charge Santa Fe Coast Lines Hospital. ALBU QUERQUE, N. MEX.

J. L., Mexican, laborer, aged 40, entered Santa Fe Hospital April 17, with fracture of left patella at lower one-third.

After remaining in bed until April 20, when the extreme effusion had somewhat subsided, on being told of the length of time necessary for repair, entailing several weeks' confinement to bed, the patient precipitately left the hospital, refusing further treatment or advice.

On May 23 he again presented himself at the hospital for the necessary certificate of discharge for reinstatement in his position. The leg had been in constant use in the interim. No effort at treatment or immobilization having been made.

In the absence of effusion, the deep sulcus separating the fragments was clearly demonstrated, the distance between the fragments being about three-quarters of an inch, with apparently a thin band of ligamentous union present. The function, however, was remarkably good, there being almost complete extension and no inconvenience in walking.

The question naturally arises, how much or how little advantage is gained by the conventional period of rest in the recumbent position, usually prescribed in the expectant or nonoperative method of treatment of fracture of the patella?

\section{EXOMPHALOS SUCCESSFULLY OPER- ATED ON.}

\section{F. HICKS, M.D.} DUNN, N. c.

Umbilical hernia occurs in three different forms:

1. The umbilical hernia of infants and young people, due to the weakening of the umbilical cicatrix, which yields before intra-abdominal pressure. This condition rarely persists until adult life and is readily amenable to treatment.

2. The umbilical hernia of adults, usually due to a pro. trusion of omentum or intestine through an opening in the linea alba either just above or below the umbilicus. This form usually requires operative treatment.

3. The congenital umbilical hernia, or exomphalos, which is an exceedingly rare condition due to the imperfect closure of the abdominal walls, as a result of which the intestine is not entirely withdrawn into the abdomen at birth, but is found in a cavity at the base of the umbilical cord, which is bulbous and enlarged. If the condition is overlooked it may be included in the ligature with which the cord is tied, causing fatal strangulation or a fecal fistula at best. If left untreated until the cord is separated, the peritoneal cavity will be laid open and septic peritonitis will ensue. The only treatment is immediate laparotomy, reduction of the intestine and closure of the umbilical opening by sutures.

Exomphalos is subdivided into different forms by some authors, the classification depending on the degree and amount of intestine protruded. The case reported in this paper was one of the most serious, the hernia being strangulated, invaginated and irreducible.

History.-The infant was assisted into this world by an old midwife, who had the good judgment, however, to tie the cord above the enlargement. Strange to say, the cord did not separate until the twelfth day, leaving the knuckle of intestine unprotected. The wise old midwife told the parents the condition was not uncommon, that she often treated them and ordered that the tumor be corered with a vaselin cloth until it "dried up." The hernia grew larger every day, but Nature came to the rescue of the infant by adhesions taking place between the outer layer of intestines and the edge of the umbilical ring, thus closing in the abdominal cavity; invagination continued to take place, however, by means of the inner coil of intestine forcing itself outward until strangulation and adhesions took place between the layers of the invaginated portion.

Consultation.-About the fifteenth day, two fecal fistulx were found, one at either end of the $\mathrm{T}$-shaped protrusion. Dr. S. P. J. Lee was called in on the seventeenth day, and requested Dr. O. L. Denning and myself to assist him operate; this we did on the eighteenth day.

operation.-On examination, the invagination was found to be irreducible and strangulated from constriction at the umbilical ring, so the adhesions at this point were broken and a laparotomy performed. About six or eight inches of the ileum were resected and the divided intestine was fixed by enterorrhaphy and simple suturing, i. e., the ends were brought together and stitched with Lembert sutures. After this the mesentery was stitched where a V-shaped portion had been removed; the intestines were replaced and the operation completed by stitching the peritoneum and closing the abdominal wall. The bowels were kept locked by opiates for six days; when the infant had a natural evacuation, and since then has had an uneventful recovery.

This case is reported on account of its rare occurrence and the almost universally fatal termination. The success attending this operation, which was done under adverse circumstances, should be an incentive to others not to give up hope as long as life exists in the patient.

\section{New Instrument}

\section{'THE COLUMBIA TISSỤE AND INTESTINAL FORCEPS. \\ I. S. STONE, M.D. \\ WASHINGTON, D. C.}

The accompanying cut shows a most satisfactory pair of forceps for general and special surgical work. They have been used at the Columbia Hospital for many years and have heretofore been made to order by instrument makers, but do not appear in any instrument catalogue nor have they ever been described, as far as I know. These forceps are valuable

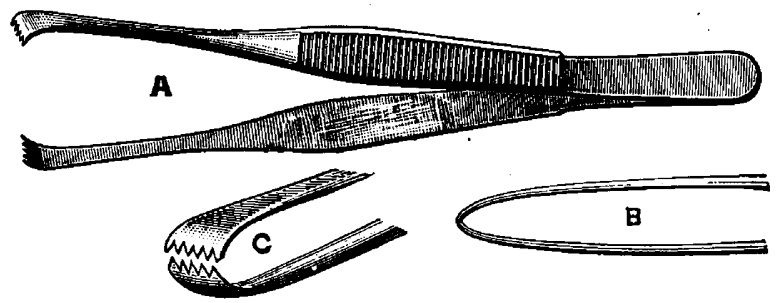

in pelvic work, especially in intestinal surgery, as they will not easily puncture the intestine as will rat-tooth forceps. In fact, they are like the delicate finger touch which permits handling without injury. The arms open widely above the teeth, allowing plenty of space for grasping sponges or larger portions of muscle or fat, or the handling of any organ within the abdomen. They are six inches in length, but longer foreeps can be made as ordered.

Apocynum Cannabinum.-In malarial conditions with torpid liver, in jaundice, in all cases where there is a sallow, yellowish skin, with foul tongue and clay-colored feces, it has a peculiarly happy effect. In fact, in all so-called bilious conditions it is almost a specific. It unlocks the secretions of the liver, secures a free discharge of bile, and becomes, if pushed, an efficient cathartic, although it is primarily an ideal cholagogue.-G. S. Chalmers, of Galesburg, Ill., in Therapeutic Gazette. 